\title{
SPATIAL STRUCTURE VARIATIONS OF PULSAR EMISSION SOURCES
}

\author{
T. V. SMIRNOVA \\ Radio Astronomy Department, Lebedev Physical Institute
}

\begin{abstract}
Diffractive scintillations from large-scale irregularities of the interstellar medium were observed for 5 strong pulsars at $102.7 \mathrm{MHz}$. The shifts of the time cross-correlation function of intensity variations at different longitudes of the profile were obtained as a function of longitude separation (phase curve). The shift is defined by the transverse separation of the pulsar emission sources. The maximum values of the spatial separation are: $1-2 \times 10^{8} \mathrm{~cm}$. We show that the phase curves are inconsistent with the dipole model of the magnetosphere and change from day to day not only in amplitude, but also in direction.
\end{abstract}

\section{Introduction}

Spatial structure of pulsar emission sources can be evaluated from the interstellar scintillation analysis of space-separated sources (Cordes, Weisberg, and Boriakoff 1983, Wolszczan and Cordes 1987, Smirnova and Shishov 1989). The regime of multiple ray interference from large scale irregularities is realized at meter wavelengths, and it was shown by Smirnova and Shishov 1989 that the value of the spatial separation of sources can be obtained from the analysis of the time cross-correlation function shift defined from the intensity variations at different pulse longitudes. Here we report the interstellar scintillation observations of 5 pulsars and their analysis.

\section{Observations and data process- ing}

Observations of 5 strong pulsars: PSR $0823+26$, $0834+06,1133+16,1237+25$ and $1919+21$ were made on November 1988 and January-May 1989 at $102.7 \mathrm{MHz}$ with the BSA telescope at Pushchino, receiving only one linear!y polarized component of the emission. The observation series was carried out for a total duration of 14 days for each pulsar. The time duration of one measurement was $3.5 / \cos (\delta)$ minutes, where $\delta$ is the source declination. A 32 channel receiver with $5-\mathrm{kHz}$ bandwidth per channel, and a detector time constant of $3 \mathrm{~ms}$ was used. Individual pulses were recorded on magnetic tape for all 32 channels with a sampling rate of $2.432 \mathrm{~ms}$ in a synchronized window of $156 \mathrm{~ms}$. The 'off pulse' part of the window was used for calibration.

The characteristic scales of time and frequency scintillation structure, $\Delta t_{\mathrm{d}}$ and $\Delta \nu_{\mathrm{d}}$, were determined as the half-widths at half-maxima of the corresponding correlation functions. To obtain $\Delta t_{\mathrm{d}}$ we computed the dependence of the correlation coefficient from a time between the pulse spectrum in the middle of a record, as a reference, and all the following and preceding spectra. The pulse spectra were taken at the longitude of maximum amplitude of the integrated profile. To exclude the intrinsic pulse-to-pulse intensity variations, spectra were averaged for 5 pulses before computing the CCF for all pulsars, except for PSR $1133+16$. To define $\Delta \nu_{\mathrm{d}}$, we chose pairs of strong pulses and computed a mean CCF from neighboring spectra on the maximum amplitude longitude of the mean profile. The scale $\Delta \nu_{1 / 2}$, obtained from the CCF directly, must be corrected for the finite bandwidth of our receiver, which smooths the real frequency structure. At our frequency the scintillations are strong and saturated, so the modulation index must be 1. We computed the mean modulation index, $m$, from 10-pulse averaged spectra for all the observations and used the dependence of $m$ on $K$, the ratio of receiver bandwidth to $\Delta \nu_{\mathrm{d}}$ (Lee 1976), that is $\Delta \nu_{\mathrm{d}}=\Delta \nu_{1 / 2} / K$. Note that the influence of polarization on intensity variations in our $160 \mathrm{kHz}$ bandwidth is small.

\section{Results}

The mean values of the time and frequency scales, $\Delta t_{\mathrm{d}}, \Delta \nu_{\mathrm{d}}$ and $\Delta \nu_{1 / 2}$, of scintillations and the modulation index, $m$, are given in table 1 .

Extrapolation of data obtained at $326 \mathrm{MHz}$ (Balasubramanian and Krishnamohan 1985) to our frequency according to $\Delta t_{\mathrm{d}} \sim f$ and $\Delta \nu_{\mathrm{d}} \sim f^{4}$ (for a Gaussian spectrum of irregularities) gives values of $\Delta t_{\mathrm{d}}^{0}$ and $\Delta \nu_{\mathrm{d}}^{0}$ as given in table 1 . As can be seen for PSR $0823+26,1237+25$, and $1919+21$ $\Delta t_{\mathrm{d}} \cong \Delta t_{\mathrm{d}}^{0}$, but for PSR 0834+06 and $1133+16$ the time scales are significantly smaller. The measured modulation index is less than 1 for all pulsars, so we must do a correction for the smooth- 
Table 1 Mean values of time and frequency scales of scintillations and modulation index

\begin{tabular}{|ccccccc|}
\hline PSR & $\begin{array}{c}\Delta t_{d}^{0} \\
(\mathrm{~s})\end{array}$ & $\begin{array}{c}\Delta t_{d} \\
(\mathrm{~s})\end{array}$ & $m$ & $\begin{array}{c}\Delta \nu_{1 / 2} \\
(\mathrm{kHz})\end{array}$ & $\Delta \nu_{d}$ & $\Delta \nu_{d}^{0}$ \\
\hline $0823+26$ & 31 & 35 & $0.56 \pm 0.06$ & 3.5 & 1 & 1.7 \\
$0834+06$ & 64 & 65 & $0.5 \pm 0.1$ & 3.7 & 0.73 & 3.7 \\
$1133+16$ & 56 & 7 & $0.5 \pm 0.1$ & 5 & 1.5 & 7 \\
$1237+25$ & 88 & 32 & $0.73 \pm 0.14$ & 5 & 3.3 & 5.8 \\
$1919+21$ & 68 & 70 & $0.7 \pm 0.1$ & 7.7 & 4.8 & 3.3 \\
\hline
\end{tabular}

PSR $1133+16$
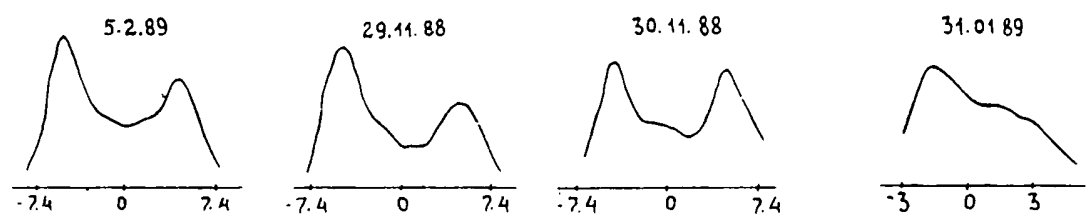

PSR $0834+06$
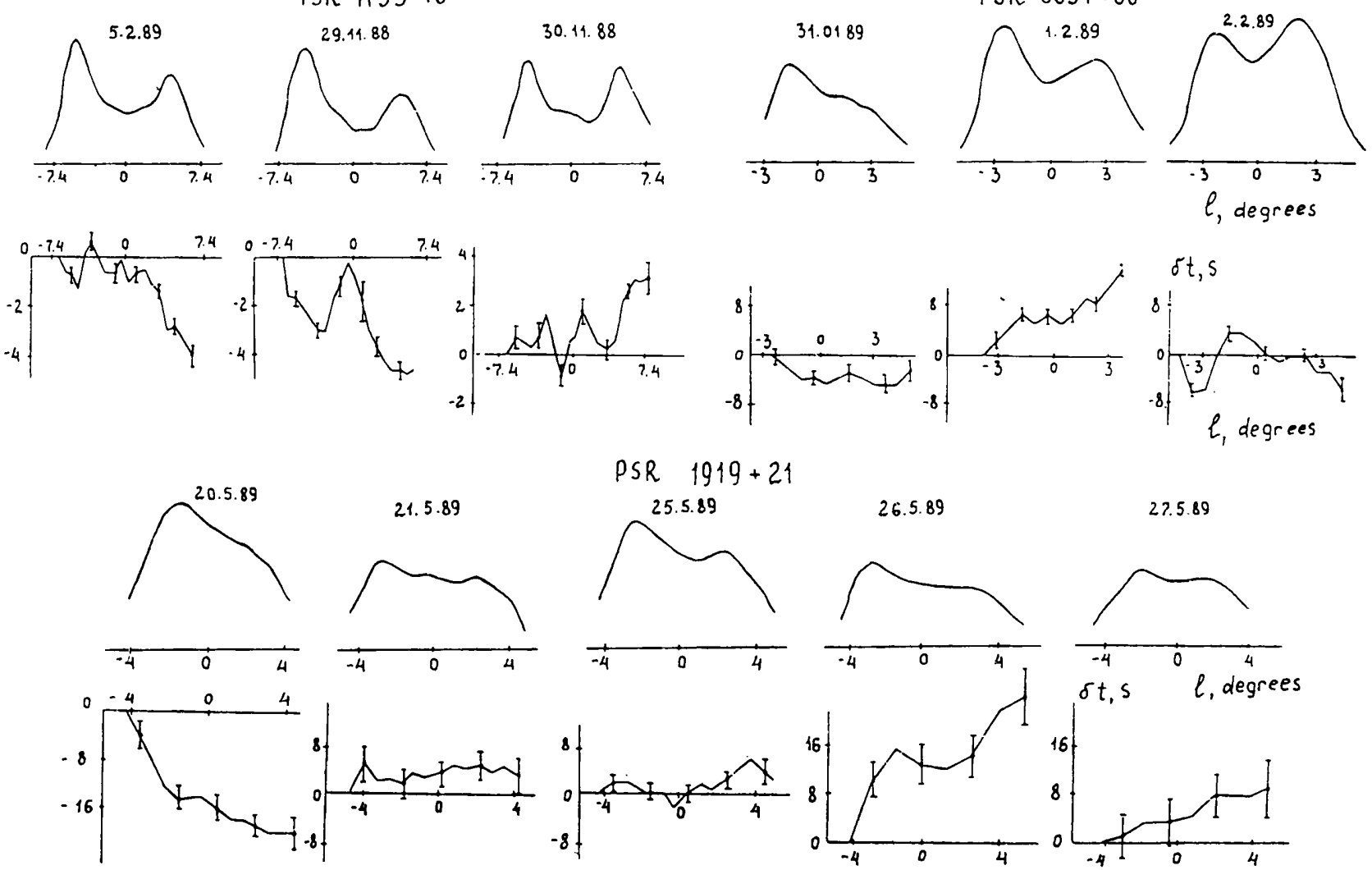

Figure 1 The average profiles and phase curves for three pulsars.

ing by our finite bandwidth, as mentioned above. The frequency scale $\Delta \nu_{\mathrm{d}}<\Delta \nu_{\mathrm{d}}^{0}$ for all pulsars except PSR 1919+21. Assuming a power spectrum for the electron-density irregularities, we have $\Delta t_{\mathrm{d}} \sim f^{1.2}$ and $\Delta \nu_{\mathrm{d}} \sim f^{4.4}$, which gives a factor of 1.6 decrease in $\Delta \nu_{d}^{0}$. So the frequency scales of PSR $0823+26$ and $1237+25$ can agree with the the extrapolated values, but for PSR $1133+16$ and $0834+06$ the difference is substantial. The time scales of PSR $1133+16$ and $1237+25$ cannot agree with extrapolated values as well. The difference between the measured and extrapolated scales may be because at our frequency diffractive scintillations occur from large inhomogenities $\left(10^{13}-10^{14} \mathrm{~cm}\right)$, while diffraction at $f \geq 300 \mathrm{MHz}$ results from small scale irregularities $\left(10^{10}-10^{11} \mathrm{~cm}\right)$.

Sometimes in the dynamic spectra of pulsars some details with approximately equal spacing can be seen which exist during times $t \leq \Delta t_{\mathrm{d}}$. The periodic intensity modulation for frequency, caused by refraction in the interstellar medium, must have a duration of $t \gg \Delta t_{\mathrm{d}}$, so we concluded that there was no refraction at $102 \mathrm{MHz}$. We did a cross correlation analysis of 10-pulse averaged spectra at the longitudes of the leading and trailing edge of the pulse of PSR 1919+21 in different parts of the data obtained on 25 May 1989. In this record the spectra had several periodic details with spacing $\sim 25 \mathrm{kHz}$ for part of the time. We obtained the CCF shift of $\delta=0.63 \pm 0.3 \mathrm{kHz}$ (with 'periodicity' present) and $\delta=1.26 \pm 0.4 \mathrm{kHz}$ (without the 'periodicity').

To obtain the transverse separation of emission regions in the pulsar magnetosphere, we computed a cross-correlation function of time intensity vari- 
Table 2 Ratio of emission altitude to the light cylinder radius

\begin{tabular}{|cccccc|}
\hline PSR & $\begin{array}{c}V_{\perp} \times 10^{5} \\
(\mathrm{~cm} / \mathrm{s})\end{array}$ & $\begin{array}{c}\delta t \\
(\mathrm{~s})\end{array}$ & $\begin{array}{c}\rho \times 10^{8} \\
(\mathrm{~cm})\end{array}$ & $\begin{array}{c}\rho \times 10^{8} \\
(\mathrm{~cm})\end{array}$ & $h / r_{L C}$ \\
\hline $0823+26$ & 365 & $<3.2$ & $<1.2$ & $<1.2$ & $<1.3$ \\
$0834+06$ & 104 & 7 & 0.73 & 1.5 & 0.46 \\
$1133+16$ & 264 & 2 & 0.53 & 1.2 & 0.17 \\
$1919+21$ & 87 & 12 & 1 & 2.1 & 0.59 \\
\hline
\end{tabular}

ations, $I(t)$, with the leading part of the profile used as a reference, and all following longitudes of the integrated pulse profile at 2.432 -ms intervals. The CCF shift is determined as the position of the center of the half-width at half-maximum of the CCF. To exclude the intrinsic pulse-to-pulse intensity variations, smoothing of $I(t)$ by 6 points was done before computing the CCF ( 4 points for PSR 1133+16). The average profiles and phase curves (the dependence of CCF shift, $\delta t$, on longitude) on different days for 3 pulsars are shown in figure 1. PSR $1237+25$, though it has strong pulse-to-pulse modulation, is the weakest of these pulsars, so we could not obtain a phase curve for it. In figure 1 we can see that the phase curves change from day to day for all pulsars, not only in amplitude, but also in direction. The time scale of change is less than 1 day. Large CCF shifts, much larger than the measurement errors, were observed one day (20 May 1989, PSR 1919+21); they became small $(\delta t \leq 2 \sigma)$ on the next day, and on 26 May 1989, the direction of the shift reversed. The direction was considered positive if $I(t)$ on the trailing longitudes passed ahead of $I(t)$ on the leading ones. The phase curves for all pulsars had a similar behavior: a non-monotonic increase with growth of the longitude separation and a flattening near the zero longitude. This phase behavior contradicts the prediction of the dipole model of magnetosphere [where $\delta t(l)$ must be monotonic] and suggests that the spatial structure of the emission sources changes in times of less than one day. The transverse displacement of sources, $\rho$, defined by $\rho=\delta t V_{\perp}$, where the velocity, $V_{\perp}$, was taken from the measurements of Lyne, Anderson and Salter (1982), and Gullahorn and Rankin (1976). The mean values of the CCF shift $(\delta t), \rho$ and the observed maximum transverse separation between the emission regions, $\rho_{\mathrm{m}}$, are given in table 2. For PSR $0823+26$ the maximum shift was $1.2 \pm 2 \mathrm{~s}$. Though a dipole model is not consistent with our phase curves, we assigned the ratio of emission altitude to the light cylinder radius, $h / r_{\mathrm{LC}}$, for this model in table 2 . 